\title{
For a fixed Turaev shadow Jones-Vassiliev invariants depend polynomially on the gleams
}

Urs Burri

Abstract. We use Turaev's technique of shadows and gleams to parametrize the set of all knots in $S^{3}$ with the same Hopf projection. We show that the Vassiliev invariants arising from the Jones polynomial $J_{t}(K)$ are polynomials in the gleams, i.e., for $n \geq 2$, the $n$-th order Vassiliev invariant $u_{n}$, defined by $J_{e^{x}}(K)=\sum_{n=0}^{\infty} u_{n}(K) x^{n}$, is a polynomial of degree $2 n$ in the gleams.

Mathematics Subject Classification (1991). 57M25.

Keywords. Vassiliev invariants, shadows, gleams, knots, Jones polynomial.

\section{Introduction}

In Turaev's seminal paper Shadow links and face models of statistical mechanics [T] knots and links in $S^{1}$-fibred 3-manifolds over oriented surfaces are presented by their Turaev shadows and gleams. Using these ideas we give an explicit parametrization $\mathcal{K}$, see Section 1 , of the set of all knots in the 3 -sphere $S^{3}$ which project under the Hopf map onto a fixed Turaev shadow $s$ on the two-sphere. Let $e$ be the number of double points of the Turaev shadow $s$. The map

$$
\mathcal{K}: \mathbb{Z}^{e} \times \mathbb{Z} \longrightarrow\left\{K \subset S^{3} \mid K \text { knot with generic Hopf projection } s\right\}
$$

may also be viewed as a suitable parametrization of the lattice of admissible gleams. In Theorem 3.3 we give an explicit formula for the second order Vassiliev invariant $v_{2}$. The map $v_{2} \circ \mathcal{K}: \mathbb{Z}^{e} \times \mathbb{Z} \longrightarrow \mathbb{Z}$ is a polynomial of degree 4 given by

$$
\begin{aligned}
v_{2} \circ \mathcal{K}\left(x_{1}, \ldots, x_{e}, x_{f}\right)=\sum_{i=1}^{e}\left(\frac{1}{2}\left(\alpha_{i}+\delta_{i} x_{f}\right)\left(x_{i}^{2}-x_{i}\right)-\frac{1}{6} x_{i}\left(2 x_{i}^{2}-3 x_{i}+1\right)+\mu_{i} x_{i}\right) \\
+\sum_{1 \leq i<j \leq e} c_{i j} x_{i} x_{j}+\frac{1}{24} x_{f}\left(x_{f}-1\right)\left(x_{f}+1\right)\left(x_{f}+2\right)+v_{2}(\mathcal{K}(0, \ldots, 0,0))
\end{aligned}
$$


where the coefficients are easily computable from $s$. As a by-product we obtain a formula for $v_{2}$ for standard knot diagrams on $\mathbb{R}^{2}$. The maybe best known Vassiliev invariants are related to the Jones polynomial $J_{t}(K) \in \mathbb{Z}\left[t, t^{-1}\right]$ of a knot $K$ by expanding this Laurent polynomial, after the substitution $t=e^{x}$, into the power series $\sum_{n=0}^{\infty} u_{n}(K) x^{n}$. The main result of this paper is to give a constructive proof that the $n$-th order Vassiliev invariant $u_{n}$ is a polynomial of degree $2 n$ in the gleams.

Theorem 3.6. The function

$$
u_{n} \circ \mathcal{K}: \mathbb{Z}^{e} \times \mathbb{Z} \longrightarrow \mathbb{Q}
$$

is a polynomial of degree $2 n$.

The first version of this paper was written in March '95. In June '95 two new results were obtained. Goussarov $[\mathrm{G}]$ showed that $n$-th order Vassiliev invariants are polynomials of degree $\leq 2 n$ in the gleams. This proved a conjecture by $\mathrm{O}$. Viro in 1990 that Vassiliev invariants depend polynomially on the gleams. Independently Shumakovitch $[\mathrm{S}]$ found a shadow formula for $v_{2}$ with very interesting coefficients.

\section{The parametrization $\mathrm{K}$}

In this section we give an explicit parametrization $\mathcal{K}$ of the set of all knots in the 3 -sphere $S^{3}$ which project under the Hopf map onto a fixed Turaev shadow $s$ on the two-sphere. We identify $S^{3}$ with the unit sphere in $\mathbb{C}^{2}$ and the Hopf fibration is induced by the intersection with complex lines through the center. We denote the Hopf map by $H$. First we have to fix some additional data. Let $s$ be the image of a generic immersion from $S^{1}$ to $S^{2}$, the fixed Turaev shadow. Generic means that the curve $s$ has no triple points and no selftangency points. The closure of a connected component of the complement of the curve $s$ is called region. The number of double points $e$ and the number of regions $r$ of the curve $s$ are related by $r=e+2$. After having fixed a region and an interior point, say $\infty$, of this region, we identify $S^{2}$ with $\mathbb{R}^{2} \cup\{\infty\}$. We fix a disc $D \subset \mathbb{R}^{2}$ which contains $s$. In the solid torus $H^{-1}(D)$ we fix a normal cylinder $N$, homeomorphic to $D \times[0, \pi]$ and such that the Hopf fibres either do not intersect this cylinder or intersect $N$ in vertical segments $d \times[0, \pi], d \in D$. Knots in $N$ which project onto $s$ can be presented by standard knot diagrams with the usual over and under crossing information. For technical reasons we also fix an extra point $P_{f}$ on $s$ which is incident to the unbounded region and orient $s$ in such a way that the unbounded region lies on the right hand side when we pass through $P_{f}$. In Figure 1, on the left hand side, we see a piece of a knot, passing through $H^{-1}$ (small disc) and intersecting the oriented Hopf fibre over $P$. The processes of positive and negative fibre fusions, respectively, are indicated in the same figure. 

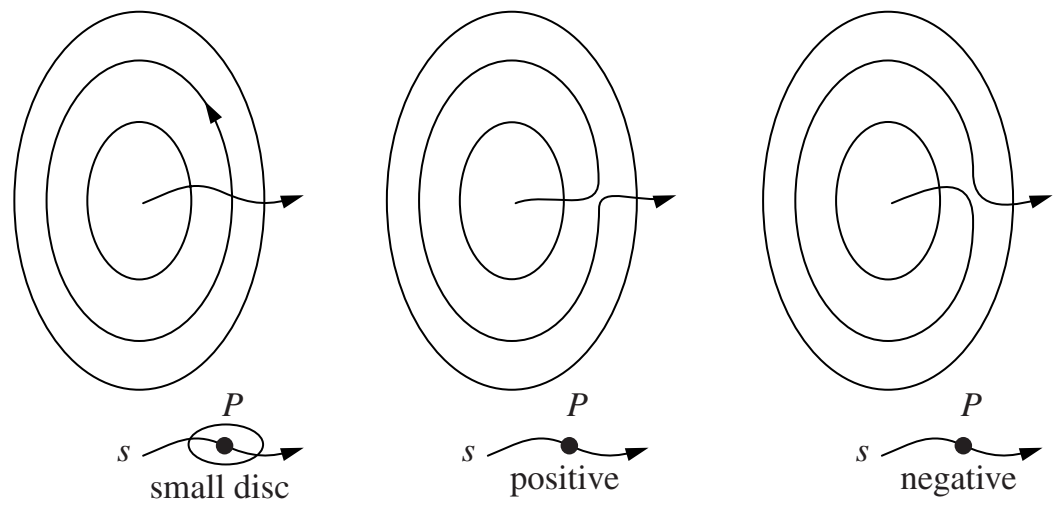

Figure 1

The point $P$ we marked on $s$ is also called blowing-up point. Given $n \in \mathbb{Z}$, to perform $n$ fibre fusions in a blowing-up point $P$ means to choose $|n|$ different points on $s$, very close to $P$, and to make for each of these points a positive or negative fibre fusion if $n$ is positive or negative, respectively. Now we can define the map

$$
\mathcal{K}: \mathbb{Z}^{e} \times \mathbb{Z} \longrightarrow\left\{K \subset S^{3} \mid K \text { knot with generic Hopf projection } s\right\} .
$$

Let $\mathcal{K}(0, \ldots, 0,0)$ be the knot in $N$ with negative crossings only. Now take this knot and perform the fibre fusions shown in Figure 2 near each double point $P_{i}$, $i \in\{1, \ldots, e\}$ and in $P_{f}$ to obtain the $\operatorname{knot} \mathcal{K}\left(x_{1}, \ldots, x_{e}, x_{f}\right)$.
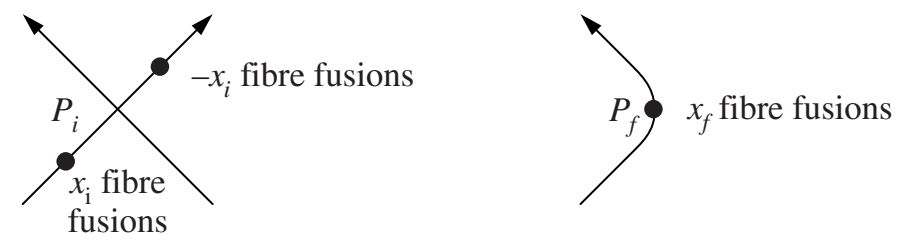

Figure 2

Note that this knot is well defined only up to vertical isotopy, i.e., isotopy where each point of the knot moves along the fibre. We will see in the next section that, up to vertical isotopy, the map $\mathcal{K}$ is surjective. This could also be shown directly using the vertical isotopies indicated in Figure 3.

Example. In Figure 4 we see the trivial curve $s$ with no double point and the knot $\mathcal{K}(3)$ which projects under the Hopf map onto $s$. The sequence of knots $x_{f} \longmapsto \mathcal{K}\left(x_{f}\right)$ will be very important later. For $x_{f} \in\{-2,-1,0,1\}$ we obtain the 

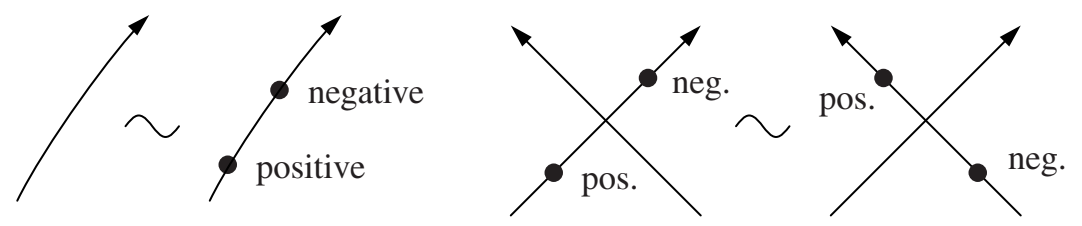

Figure 3

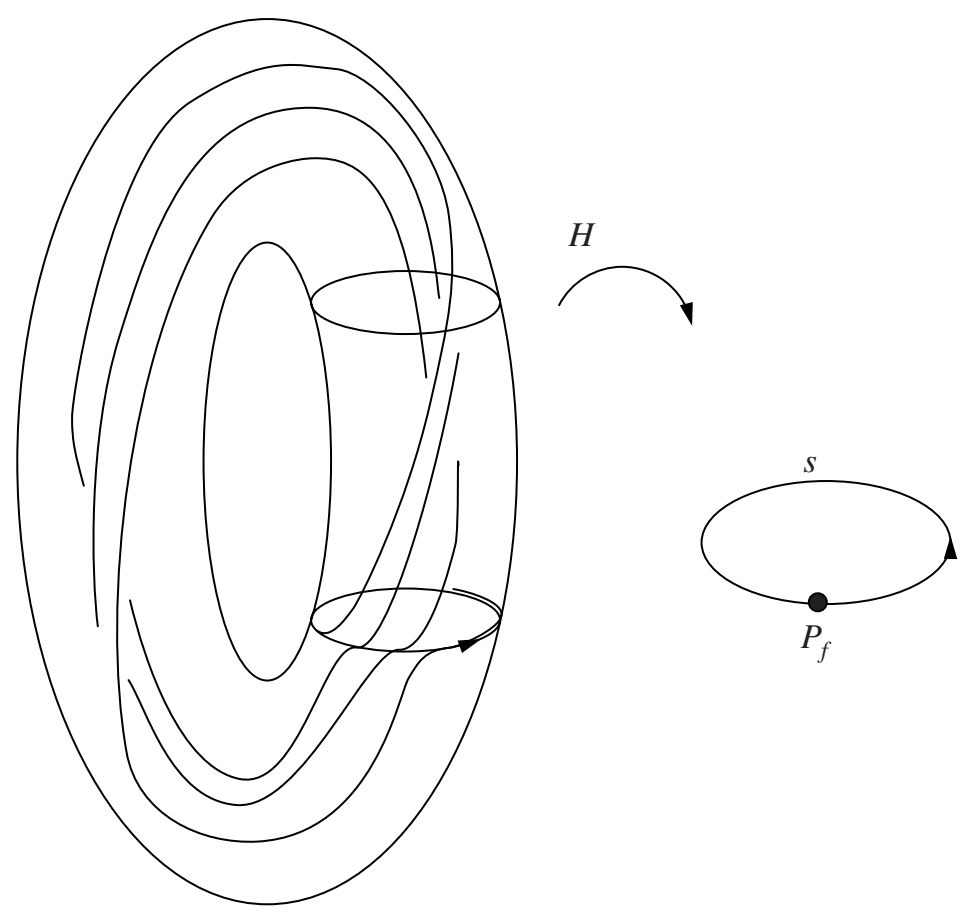

Figure 4

trivial knot, and for $x_{f} \geq 2, \mathcal{K}\left(x_{f}\right)$ is the torus knot $\mathcal{T}\left(x_{f}, x_{f}+1\right)$ which is isotopic to $\mathcal{K}\left(-1-x_{f}\right)$.

The map $\mathcal{K}$ has several important properties. The knots $\mathcal{K}\left(\ldots, x_{i}+1, \ldots, x_{f}\right)$ and $\mathcal{K}\left(\ldots, x_{i}, \ldots, x_{f}\right)$ differ by a positive crossing change. After the vertical isotopy indicated in Figure 5 it is sufficient to consider what happens inside $T_{i}:=$ $H^{-1}\left(D_{i}\right)$. We see that the loop can be moved back to the normal cylinder by a vertical isotopy.

This also shows that if $x_{i} \in\{0,1\}$ for all $i \in\{1, \ldots, e\}$, then, after a vertical isotopy, $\mathcal{K}\left(x_{1}, \ldots, x_{e}, 0\right)$ lies in $N$ and $\mathcal{K}\left(x_{1}, \ldots, x_{e}, x_{f}\right)=\mathcal{K}\left(x_{1}, \ldots, x_{e}, 0\right) \# \mathcal{K}\left(x_{f}\right)$. For the sake of simplicity, we denote by $\mathcal{K}\left(\ldots, x_{i}+\frac{1}{2}, \ldots, x_{f}\right)$ the singular knot 

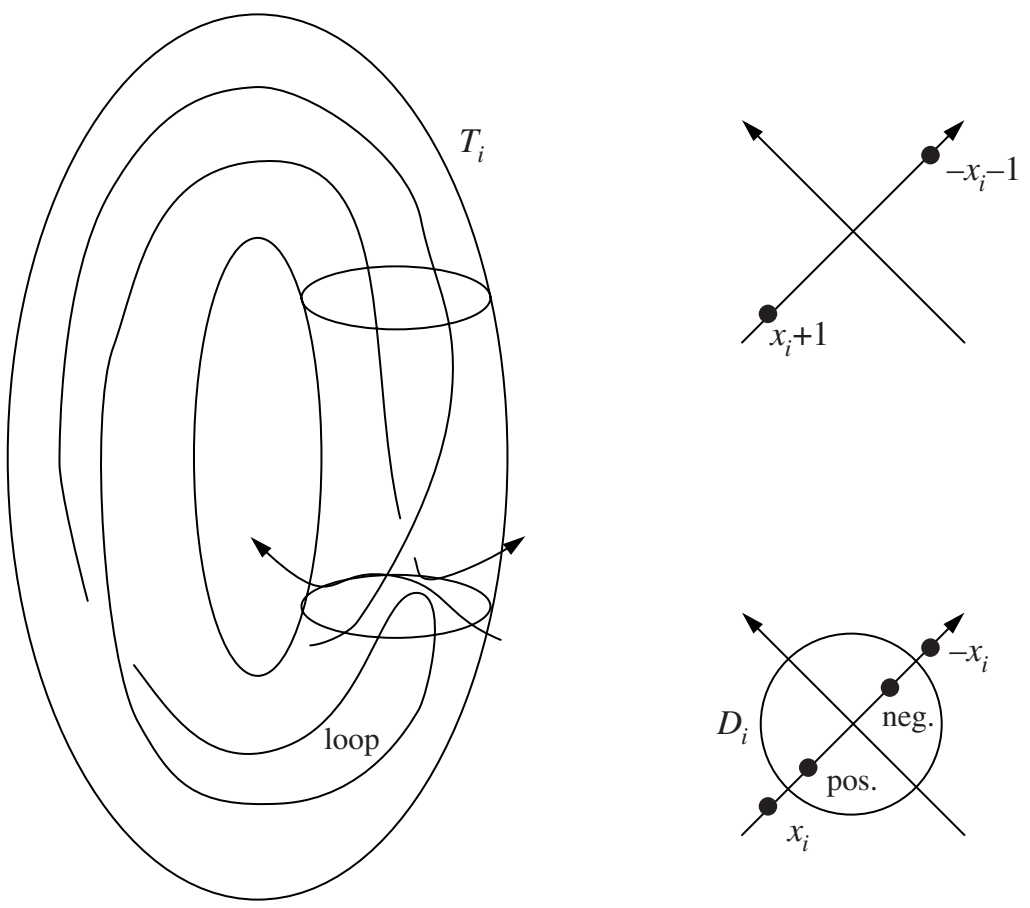

Figure 5

with positive and negative resolution $\mathcal{K}\left(\ldots, x_{i}+1, \ldots, x_{f}\right)$ and $\mathcal{K}\left(\ldots, x_{i}, \ldots, x_{f}\right)$, respectively.

\section{Turaev's construction}

We shall only describe Turaev's construction for the special case of links in $S^{3}$ which project under the Hopf map $H$ to $S^{2}$. For generalizations and details we refer to [T]. We consider a link $L \subset S^{3}$ which generically projects under $H$ to $S^{2}$. We can vertically isotop this link to $L^{\prime}$ such that any two pre-images of any double point lie opposite in the Hopf fibre. Let $q: S^{3} \longrightarrow \mathbb{R P}^{3}$ be the canonical two-sheeted covering and let $h^{\prime}$ be the circle bundle induced by the commutative diagram $H=h^{\prime} \circ q$. The graph $q\left(L^{\prime}\right)$ is mapped bijectively onto $H(L)$ by $h^{\prime}$. The inverse bijection $r: H(L) \longrightarrow q\left(L^{\prime}\right)$ is a section of $h^{\prime}$ over $H(L)$. The restriction of $h^{\prime}$ to a region $X$ is a trivial bundle. We identify it with the projection $S^{1} \times X \longrightarrow X$. Denote by $\alpha_{X}$ the degree of the composition of $\left.r\right|_{\partial X}: \partial X \longrightarrow S^{1} \times X$ with the projection $S^{1} \times X \longrightarrow S^{1}$. This is the obstruction to extend $\left.r\right|_{\partial X}$ over $X$. Let $\beta_{X}$ be the number of corners (counted with multiplicities) of the region $X$. The gleam of $X$ is defined to be $\frac{\beta_{X}-\alpha_{X}}{2}$. It is an integer, independent of the choice 
of $L^{\prime}$ and the trivialisation of $h^{\prime}$. The total gleam is defined by the sum of the gleams over all regions, minus twice the number of double points. Each double point contributes to 4 corners, so the total gleam equals $-\frac{1}{2} \sum_{X} \alpha_{X}$. The sum of the obstructions $\alpha_{X}$ can be identified with $\chi\left(h^{\prime}\right)=2 \chi(H)=-2$, where $\chi(\xi)$ is the Euler number of the two dimensional real vector bundle associated to the oriented circle bundle $\xi$. The Turaev shadow $H(L)$ together with its (integer) gleams is called the shadow of $L$ and denoted by $S(L)$, its total gleam equals one. We call two shadows equivalent if they can be transformed to each other by a finite number of shadow moves, see Figure 6 . The local gleams $x, y, z, \ldots$ are arbitrary integers, but 0,1 and 2 play a distinguished role.
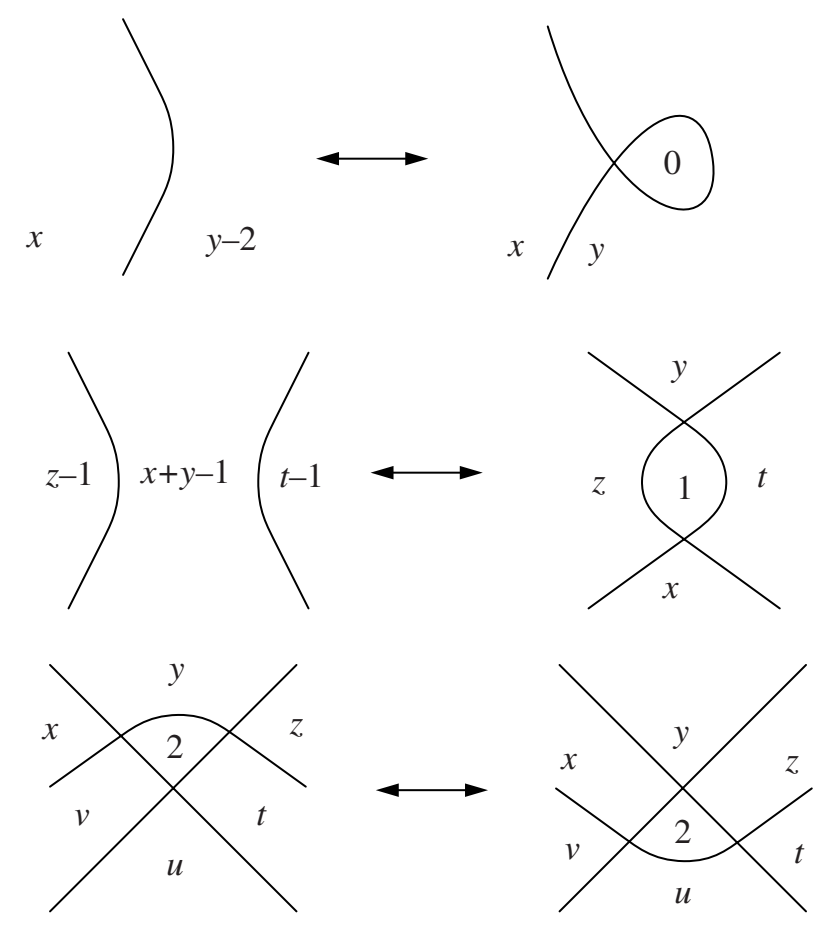

Figure 6

Theorem 2.1 (Turaev [T]). Two generic links $L_{1}, L_{2} \subset S^{3}$ are ambient isotopic if and only if their shadows $S\left(L_{1}\right), S\left(L_{2}\right)$ are equivalent. The map $L \longmapsto S(L)$ induces a bijective correspondence between the set of isotopy types of links in $S^{3}$ and equivalence classes of shadows.

Note that there also exists an oriented version for this theorem, where the links and the corresponding Turaev shadows are oriented. The orientation of the fixed Turaev shadow $s$ was chosen only for technical reasons. The proof of 
this theorem shows that the set of links, which has as Hopf projection the same fixed generic collection of loops, is parametrized by the gleams. For the gleams there is only one restriction, the total gleam has to be equal to one. For the case of knots with Turaev shadow $s$, this lattice of admissible gleams is given by $\left\{\left(g_{1}, \ldots, g_{r}\right) \in \mathbb{Z}^{r} \mid \sum_{j=1}^{r} g_{j}-2 e=1\right\}$.

Theorem 2.2. The map

$$
\mathcal{K}: \mathbb{Z}^{e} \times \mathbb{Z} \longrightarrow\left\{K \subset S^{3} \mid K \text { knot with generic Hopf projection } s\right\}
$$

described above is surjective up to vertical isotopy.

Before we prove this theorem, let us study the gleams of $\mathcal{K}\left(x_{1}, \ldots, x_{e}, x_{f}\right)$. The local gleams of $\mathcal{K}(0, \ldots, 0,0)$ are given by the following rule:

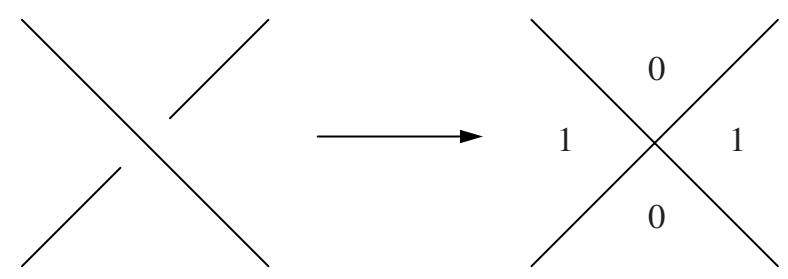

Figure 7

To obtain the gleam of a region we have to sum up these local gleams and we have to add one to the gleam of the unbounded region. Firstly we may assume that the normal cylinder $N$ is exactly half of $H^{-1}(D)$ and secondly that for a knot in $N$ the lower branch near a crossing point lies in the meridional disc $D \times\{0\}$ and the upper branch in $D \times\{\pi\}$. Then everything follows directly from the definition of the gleams. The local gleams of fibre fusions are shown on the left hand side of Figure 8. On the right hand side, we see the local gleams of the fibre fusions near the double point $P_{i}$.

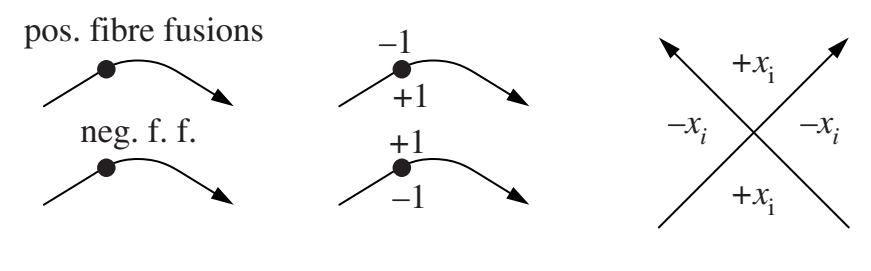

Figure 8

Proof of Theorem 2.2. We observe that we can realize all admissible gleams of $s$ using the following trick: 


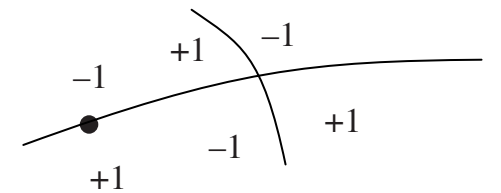

$+1$

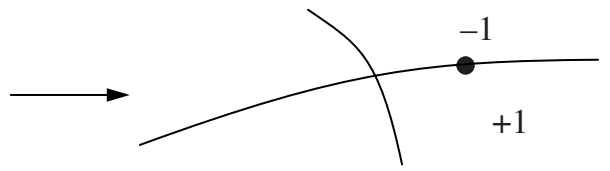

Figure 9

Moreover, for a fixed Turaev shadow $s$, the gleam of a knot of a region minus the gleam of $\mathcal{K}(0, \ldots, 0,0)$ of the same region is a $\mathbb{Z}$-linear combination of $x_{1}, \ldots, x_{e}, x_{f}$. The transition matrix depends on $s$ and on the choices of $\infty$ and $P_{f}$. This shows that a knot invariant $I$ is a polynomial of degree $m$ in the gleams if and only if $I \circ \mathcal{K}$ is a polynomial of degree $m$.

\section{The main results}

In this section we examine how knot invariants depend on the gleams. Given a $\mathcal{G}$-valued knot invariant $I$, where $\mathcal{G}$ is an abelian group, what does the map $I \circ \mathcal{K}: \mathbb{Z}^{e} \times \mathbb{Z} \longrightarrow \mathcal{G}$ look like?

As we will also deal with derivatives, i.e., finite differences, of functions defined on the lattice $\mathbb{Z}^{e} \times \mathbb{Z}$, we introduce the notation $\Delta_{x_{j}}(I \circ \mathcal{K})\left(\ldots, x_{j}, \ldots\right)$ for the difference $(I \circ \mathcal{K})\left(\ldots, x_{j}+1, \ldots\right)-(I \circ \mathcal{K})\left(\ldots, x_{j}, \ldots\right)$.

We shall use the following result:

Theorem 3.1 (Birman, Lin [B-L]). Let $K$ be a knot and let $J_{t}(K)$ be its onevariable Jones polynomial. Let $U_{x}(K)$ be obtained from $J_{t}(K)$ by replacing the variable $t$ by $e^{x}$. Using the power series expansion of $e^{x}$ to express $U_{x}(K)$ as a power series in $x$ :

$$
U_{x}(K)=\sum_{n=0}^{\infty} u_{n}(K) x^{n}
$$

Then $u_{0}(K)=1$ and $u_{n}(K), n \geq 1$ is a Vassiliev invariant of order $n$.

Example. We consider again the sequence of knots $x_{f} \mapsto \mathcal{K}\left(x_{f}\right)$. We try to examine how Jones-Vassiliev invariants depend on $x_{f}$. We use the fact that the one-variable Jones polynomial $J_{t}$ of the torus $\operatorname{knot} \mathcal{T}(p, p+1), p \geq 2$, is given by the following formula of Jones $[\mathrm{J}]$

$$
J_{t}(\mathcal{T}(p, p+1))=\frac{t^{p(p-1) / 2}\left(1-t^{p+1}-t^{p+2}+t^{2 p+1}\right)}{1-t^{2}}
$$


We calculate:

$$
J_{t}(\mathcal{T}(p, p+1))=t^{p(p-1) / 2}\left(t^{p}+\sum_{k=0}^{p-1}\left(t^{k}-t^{2 k+1}\right)\right),
$$

and get, for $p \geq 2, U_{x}(\mathcal{T}(p, p+1))=\sum_{n=0}^{\infty} u_{n}(p) x^{n}$, where

$$
u_{n}(p)=\sum_{j=0}^{n} \frac{(p(p-1))^{n-j}}{2^{n-j} j !(n-j) !}\left(\sum_{k=0}^{p} k^{j}-\sum_{k=0}^{p-1}(2 k+1)^{j}\right) .
$$

The function $u_{n}$ is a polynomial of degree $2 n$ in $p$ for $p \geq 2$. Extended to a polynomial defined on $\mathbb{Z}, u_{n}$ has the symmetry $u_{n}(p)=u_{n}(-1-p)$, i.e., $u_{n} \circ \mathcal{K}$ is a polynomial in $x_{f}$ of degree $2 n$. As expected, we have $u_{1}(p)=0$. For $u_{2}$ we get the nice expression $u_{2}(p)=-\frac{1}{8} p(p-1)(p+2)(p+1)$, and hence: $u_{2}\left(\mathcal{K}\left(x_{f}\right)\right)=$ $-\frac{1}{8} x_{f}\left(x_{f}-1\right)\left(x_{f}+1\right)\left(x_{f}+2\right)$.

Theorem 3.2. Let $v_{n}$ be a $\mathcal{G}$-valued Vassiliev invariant of order $n$. For each choice of indices with $1 \leq i_{1}<\ldots<i_{n} \leq e$ we have

$$
\Delta_{x_{i_{1}}} \Delta_{x_{i_{2}}} \ldots \Delta_{x_{i_{n}}}\left(v_{n} \circ \mathcal{K}\right)\left(x_{1}, x_{2}, \ldots, x_{e}, x_{f}\right)=c_{i_{1} i_{2} \ldots i_{n}},
$$

where $c_{i_{1} i_{2} \ldots i_{n}}$ is the value of $v_{n}$ on an n-singular knot with the same chord diagram as the curve s, where only the chords corresponding to the double points $P_{i_{1}}, \ldots, P_{i_{n}}$ are considered.

An example for $n=3$ is shown in Figure 10:
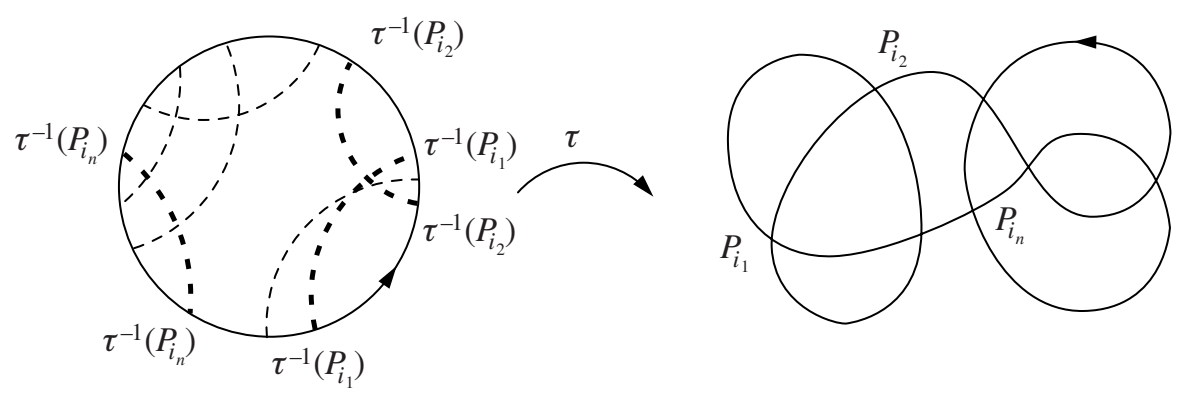

Figure 10

Proof. Let $1 \leq i_{1}<\ldots<i_{n} \leq e$. The $n$-singular knots $\mathcal{K}\left(\ldots, x_{i_{1}}+\frac{1}{2}, \ldots, x_{i_{n}}+\right.$ $\left.\frac{1}{2}, \ldots, x_{f}\right)$ have the same chord diagram for all $x_{1}, \ldots, x_{e}, x_{f} \in \mathbb{Z}$. The value of 
the $n$-th order Vassiliev invariant $v_{n}$ is constant on these singular knots. On the other hand, using the Vassiliev skein relation, we get

$$
\begin{gathered}
v_{n}\left(\mathcal{K}\left(\ldots, x_{i_{1}}+\frac{1}{2}, \ldots, x_{i_{n}}+\frac{1}{2}, \ldots, x_{f}\right)\right)= \\
\sum_{\left(\epsilon_{i_{1}}, \ldots, \epsilon_{i_{n}}\right) \in\{0,1\}^{n}}(-1)^{n-\left(\epsilon_{i_{1}}+\ldots+\epsilon_{i_{n}}\right)} v_{n}\left(\mathcal{K}\left(\ldots, x_{i_{1}}+\epsilon_{i_{1}}, \ldots, x_{i_{n}}+\epsilon_{i_{n}}, \ldots, x_{f}\right)\right)= \\
\Delta_{x_{i_{1}}} \ldots \Delta_{x_{i_{n}}}\left(v_{n} \circ \mathcal{K}\right)\left(x_{1}, x_{2}, \ldots, x_{e}, x_{f}\right) .
\end{gathered}
$$

Example. It is well known that the first order Vassiliev invariant $v_{1}$ is trivial. We want to check how strong our techniques are. We apply Theorem 3.2. It follows that for all $i \in\{1, \ldots, e\}$ we have $\Delta_{x_{i}}\left(v_{1} \circ \mathcal{K}\right)\left(x_{1}, \ldots, x_{e}, x_{f}\right)=0$. Remember that $v_{1}$ of a 1 -singular knot is zero, because the chord diagram has an isolated chord. This shows that $\left(v_{1} \circ \mathcal{K}\right)\left(x_{1}, \ldots, x_{e}, x_{f}\right)$ is a function, let us say $g$, of $x_{f}$ only. Now it is sufficient to find $x_{1}, \ldots, x_{e} \in\{0,1\}$ such that $\mathcal{K}\left(x_{1}, \ldots, x_{e}, 0\right)$ is the trivial knot. Then

$$
\mathcal{K}\left(x_{1}, \ldots, x_{e}, x_{f}\right)=(\text { trivial knot }) \# \mathcal{K}\left(x_{f}\right)
$$

which implies that $g\left(x_{f}\right)$ is determined by the values on the sequence $\mathcal{K}\left(x_{f}\right)$.

For the next theorem we need the following definition of $\mu_{i}, \delta_{i}, \alpha_{i}, c_{i j}$. Splicing the curve $s$ in $D_{i}$ as in Figure 11, we obtain two curves $A_{i}$ and $B_{i}$ and define $\mu_{i}:=-\frac{1}{2}\left|A_{i} \cap B_{i}\right|$. This number is equal to the linking number of the twocomponent link obtained by splicing $\mathcal{K}\left(0, \ldots, 0, \frac{1}{2}, 0, \ldots, 0,0\right)$, see Figure 11:

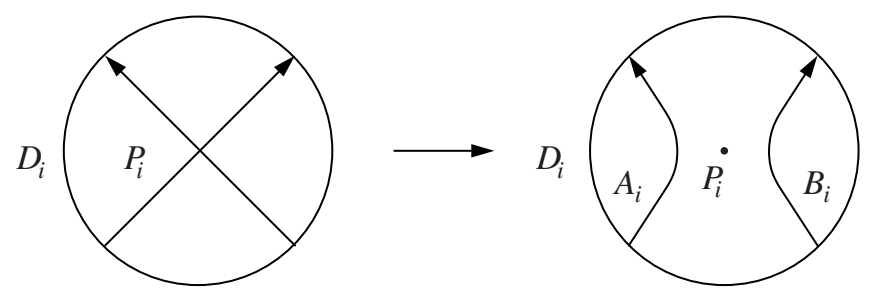

Figure 11

The integer $\delta_{i}$ is defined by $\delta_{i}:=+1$ if $P_{f} \in B_{i}$ and $\delta_{i}:=-1$ if $P_{f} \in A_{i}$. The coefficient $\alpha_{i}$ is defined to be the difference of the two winding numbers $\operatorname{ind}\left(B_{i}, P_{i}\right)$ 

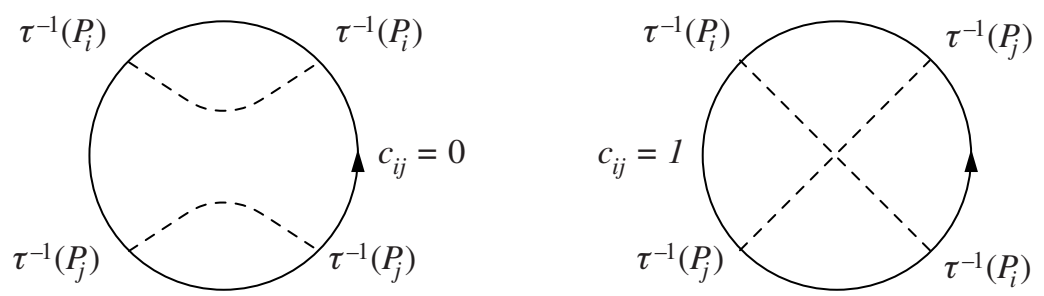

Figure 12

and $\operatorname{ind}\left(A_{i}, P_{i}\right)$. Let $\tau: S^{1} \longrightarrow S^{2}$ be a parametrization of $s$. For $i \neq j, c_{i j}$ is equal to zero or one according to Figure 12 .

Let $v_{2}$ denote the second-order Vassiliev invariant which can be identified with the coefficient of $z^{2}$ of the Alexander-Conway polynomial $\nabla_{K}(z) \in \mathbb{Z}[z]$. We know that $v_{2}=-\frac{1}{3} u_{2}$.

Theorem 3.3. The function $v_{2} \circ \mathcal{K}: \mathbb{Z}^{e} \times \mathbb{Z} \longrightarrow \mathbb{Z}$ is a polynomial of degree four given by:

$$
\begin{aligned}
& \left(v_{2} \circ \mathcal{K}\right)\left(x_{1}, x_{2}, \ldots, x_{e}, x_{f}\right)=-\frac{1}{6} \sum_{i=1}^{e} x_{i}\left(2 x_{i}^{2}-3 x_{i}+1\right)+\frac{1}{2} \sum_{i=1}^{e}\left(\alpha_{i}+\delta_{i} x_{f}\right)\left(x_{i}^{2}-x_{i}\right) \\
& +\sum_{1 \leq i<j \leq e} c_{i j} x_{i} x_{j}+\sum_{i=1}^{e} \mu_{i} x_{i}+\frac{1}{24} x_{f}\left(x_{f}-1\right)\left(x_{f}+1\right)\left(x_{f}+2\right)+v_{2}(\mathcal{K}(0, \ldots, 0,0)) .
\end{aligned}
$$

We obtain a formula for $v_{2}$ for standard knot diagrams on the plane. All knot diagrams on $\mathbb{R}^{2}$ with knot projection $s$ can be encoded using $\mathcal{K}$. We put $x_{f}=0$ and $x_{i}=0$ or 1 if the $i$-th crossing is negative or positive, respectively.

Corollary 3.4. The following formula for $v_{2}$ of standard knot diagrams holds true:

$$
v_{2}\left(\mathcal{K}\left(x_{1}, x_{2}, \ldots, x_{e}, 0\right)\right)=\sum_{1 \leq i<j \leq e} c_{i j} x_{i} x_{j}+\sum_{i=1}^{e} \mu_{i} x_{i}+v_{2}(\mathcal{K}(0, \ldots, 0,0)),
$$

where the last term can be calculated using the same formula, by choosing $x_{1}, \ldots$, $x_{e} \in\{0,1\}$ such that $\mathcal{K}\left(x_{1}, x_{2}, \ldots, x_{e}, 0\right)$ is trivial.

Corollary 3.5. The numbers $c_{i j}, \mu_{i}$ are related by

$$
\sum_{1 \leq i<j \leq e} c_{i j}+\sum_{i=1}^{e} \mu_{i}=0
$$


Proof of Corollary 3.5. The knot $\mathcal{K}(1, \ldots, 1,0)$ is the mirror image of $\mathcal{K}(0, \ldots, 0,0)$, but $v_{2}$ cannot distinguish them, i.e., $v_{2}(\mathcal{K}(1, \ldots, 1,0))=$ $v_{2}(\mathcal{K}(0, \ldots, 0,0))$ and we are done.

Proof of Theorem 3.3. We fix $i \in\{1, \ldots, e\}$ and study the function

$$
x_{i} \mapsto v_{2}\left(\mathcal{K}\left(0, \ldots, 0, x_{i}, 0, \ldots, 0,0\right)\right)
$$

We calculate

$$
\begin{gathered}
x_{i} \mapsto \Delta_{x_{i}} v_{2}\left(\mathcal{K}\left(0, \ldots, 0, x_{i}, 0, \ldots, 0,0\right)\right)= \\
v_{2}\left(\mathcal{K}\left(0, \ldots, 0, x_{i}+1,0, \ldots, 0,0\right)\right)-v_{2}\left(\mathcal{K}\left(0, \ldots, 0, x_{i}, 0, \ldots, 0,0\right)\right) .
\end{gathered}
$$

This difference is equal to the linking number of the two-component link obtained by splicing the singular knot $\mathcal{K}\left(0, \ldots, 0, x_{i}+\frac{1}{2}, 0, \ldots, 0,0\right)$ at the singular point. Since the linking number of a link is the sum of any set of crossing indices whose switchings unlink it, we can calculate this linking number explicitly. It consists of three terms. First we observe that inside $T_{i}:=H^{-1}\left(D_{i}\right)$ we have the linked fibres from the $x_{i}$ positive and negative fibre fusions we made near $P_{i}$. The first term of the linking number calculates the linking of these fibres, the second one measures how the curves $A_{i}$ and $B_{i}$ wind around $T_{i}$ and the third one does not depend on $x_{i}$. We obtain

$$
-x_{i}^{2}+\alpha_{i} x_{i}+\mu_{i}
$$

see Figure 13:

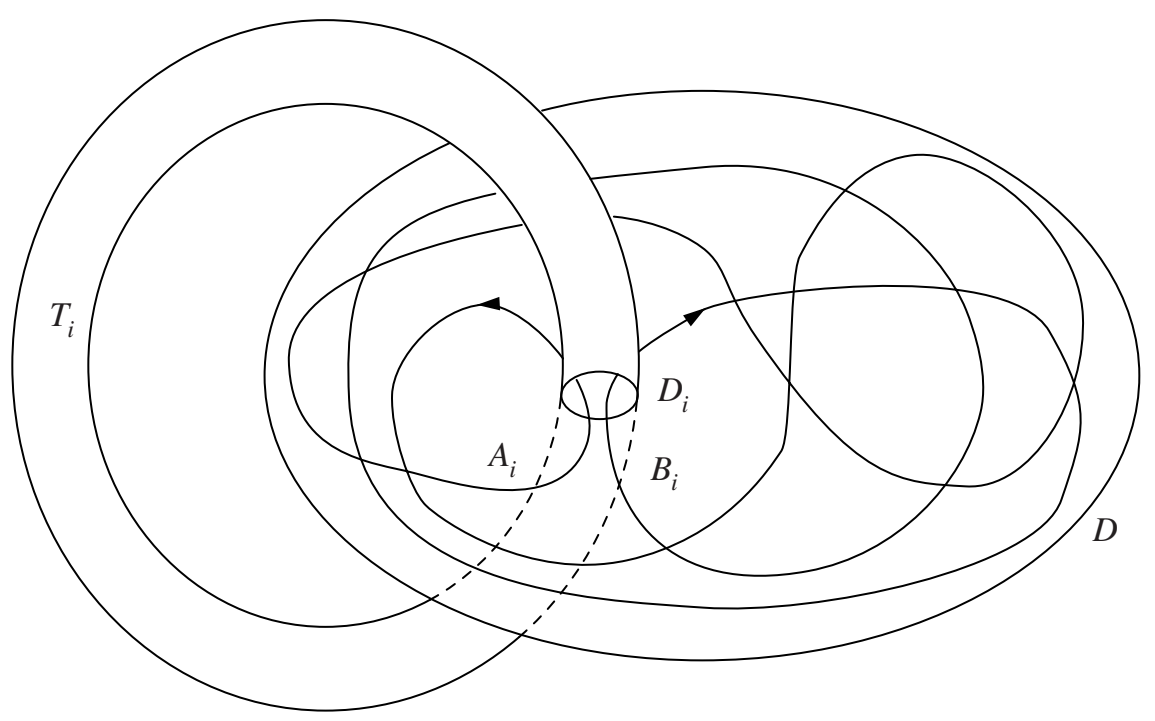

Figure 13 
Now we determine $\Delta_{x_{i}} v_{2}\left(\mathcal{K}\left(x_{1}, \ldots, x_{i}, \ldots, x_{e}, 0\right)\right)$. The quadratic term $-x_{i}^{2}$ remains unchanged, because this contribution to the linking is determined by what happens inside the solid torus $T_{i}$. The astonishing thing is that the linear term $\alpha_{i} x_{i}$ as well remains unchanged. We give two independent proofs of this fact.

The first proof: The fibres of the fibre fusions near a double point $P_{j}, j \neq i$, are linked with the fibres in $T_{i}$. But there is no contribution to the linking which grows linearly in $x_{i}$, because one half of them is run through in the positive sense and the other half in the negative sense.

The second proof: Here we use the fact that $v_{2}$ is a Vassiliev invariant. Using Theorem 3.2, we know that for $j \neq i, 1 \leq j \leq e$ we have

$$
\Delta_{x_{j}} \Delta_{x_{i}} v_{2}\left(\mathcal{K}\left(x_{1}, \ldots, x_{i}, \ldots, x_{e}, 0\right)\right)=c_{i j} .
$$

This means that the coefficient of the linear term does not depend on $x_{j}$.

The above implies

$$
\Delta_{x_{i}} v_{2}\left(\mathcal{K}\left(x_{1}, \ldots, x_{i}, \ldots, x_{e}, 0\right)\right)=-x_{i}^{2}+\alpha_{i} x_{i}+F_{i}\left(x_{1}, \ldots, \widehat{x_{i}}, \ldots, x_{e}, 0\right),
$$

where $F_{i}\left(x_{1}, \ldots, \widehat{x_{i}}, \ldots, x_{e}, 0\right)$ does not depend on $x_{i}$. Now we let $x_{f}$ run as well. The Hopf fibre over $P_{f}$ is linked with the solid torus $T_{i}$, which implies

$\Delta_{x_{i}} v_{2}\left(\mathcal{K}\left(x_{1}, \ldots, x_{i}, \ldots, x_{e}, x_{f}\right)\right)=-x_{i}^{2}+\left(\alpha_{i}+\delta_{i} x_{f}\right) x_{i}+F_{i}\left(x_{1}, \ldots, \widehat{x_{i}}, \ldots, x_{e}, x_{f}\right)$.

For $j \neq i, 1 \leq j \leq e$, the equation

$$
\Delta_{x_{j}} \Delta_{x_{i}} v_{2}\left(\mathcal{K}\left(x_{1}, \ldots, x_{i}, \ldots, x_{e}, x_{f}\right)\right)=c_{i j}
$$

implies that

$$
F_{i}\left(x_{1}, \ldots, \widehat{x_{i}}, \ldots, x_{e}, x_{f}\right)=c_{i 1} x_{1}+\ldots+\widehat{c_{i i} x_{i}}+\ldots+c_{i e} x_{e}+h_{i}\left(x_{f}\right),
$$

where $h_{i}$ depends on $x_{f}$ only. We integrate and get

$$
\begin{aligned}
& x_{i} \mapsto v_{2}\left(\mathcal{K}\left(x_{1}, \ldots, x_{i}, \ldots, x_{e}, x_{f}\right)\right)=-\frac{1}{6} x_{i}\left(2 x_{i}^{2}-3 x_{i}+1\right)+\frac{1}{2}\left(\alpha_{i}+\delta_{i} x_{f}\right)\left(x_{i}^{2}-x_{i}\right) \\
& +c_{i 1} x_{1} x_{i}+\ldots+c_{i i} \widehat{x_{i}} x_{i}+\ldots+c_{i e} x_{e} x_{i}+h_{i}\left(x_{f}\right) x_{i}+G_{i}\left(x_{1}, \ldots, \widehat{x_{i}}, \ldots, x_{e}, x_{f}\right),
\end{aligned}
$$

where $G_{i}$ is the integration constant. But this result holds for every $i \in\{1, \ldots, e\}$. We obtain

$$
\begin{gathered}
\left(v_{2} \circ \mathcal{K}\right)\left(x_{1}, x_{2}, \ldots, x_{e}, x_{f}\right)=-\frac{1}{6} \sum_{i=1}^{e} x_{i}\left(2 x_{i}^{2}-3 x_{i}+1\right)+\frac{1}{2} \sum_{i=1}^{e}\left(\alpha_{i}+\delta_{i} x_{f}\right)\left(x_{i}^{2}-x_{i}\right) \\
+\sum_{1 \leq i<j \leq e} c_{i j} x_{i} x_{j}+\sum_{i=1}^{e} h_{i}\left(x_{f}\right) x_{i}+g\left(x_{f}\right)
\end{gathered}
$$


It remains to determine $h_{i}$ and $g$. We have

$$
v_{2}\left(\mathcal{K}\left(0, \ldots, 0, x_{f}\right)\right)=g\left(x_{f}\right)
$$

Furthermore we know that $\mathcal{K}\left(0, \ldots, 0, x_{f}\right)=\mathcal{K}(0, \ldots, 0,0) \# \mathcal{K}\left(x_{f}\right)$ and that $v_{2}$ is additive under connected sum, which implies that

$$
g\left(x_{f}\right)=v_{2}(\mathcal{K}(0, \ldots, 0,0))+\frac{1}{24} x_{f}\left(x_{f}-1\right)\left(x_{f}+1\right)\left(x_{f}+2\right) .
$$

From

$$
\mathcal{K}\left(0, \ldots, 0,1,0, \ldots, 0, x_{f}\right)=\mathcal{K}(0, \ldots, 0,1,0, \ldots, 0,0) \# \mathcal{K}\left(x_{f}\right)
$$

and

$$
v_{2}\left(\mathcal{K}\left(0, \ldots, 0,1,0, \ldots, 0, x_{f}\right)\right)=h_{i}\left(x_{f}\right)+g\left(x_{f}\right),
$$

we find

$$
h_{i}\left(x_{f}\right)=v_{2}(\mathcal{K}(0, \ldots, 0,1,0, \ldots, 0,0))-v_{2}(\mathcal{K}(0, \ldots, 0,0))=\mu_{i}
$$

Theorem 3.6. Let us define $u_{n}$ as in Theorem 3.1. The function

$$
u_{n} \circ \mathcal{K}: \mathbb{Z}^{e} \times \mathbb{Z} \longrightarrow \mathbb{Q}
$$

is a polynomial of degree $2 n$.

Proof. The proof is by induction. We need the following result:

Proposition 3.7 (Birman, Lin [B-L]). Let $K_{+}, K_{-}, K_{\infty}$ be knots which are defined by immersions which agree everywhere except near a single crossing. Let $\lambda$ be the linking number of the 2 -component link $K_{0}$. Then for every $n \geq 2$ the following formula holds:

$$
\begin{aligned}
u_{n}\left(K_{+}\right)- & u_{n}\left(K_{-}\right)=u_{n-1}\left(K_{-}\right)-u_{n-1}\left(K_{\infty}\right)+\frac{1+(3 \lambda)^{n}-(3 \lambda+1)^{n}}{n !} \\
& +\sum_{j=2}^{n-2} \frac{u_{j}\left(K_{-}\right)+u_{j}\left(K_{\infty}\right)\left[(3 \lambda)^{n-j}-(3 \lambda+1)^{n-j}\right]}{(n-j) !}
\end{aligned}
$$




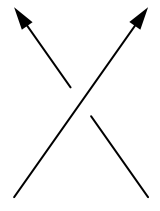

$K_{+}$

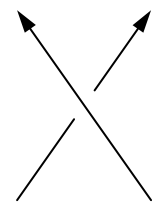

$K_{-}$

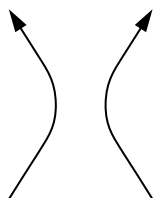

$K_{0}$

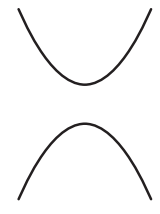

$K_{\infty}$

Figure 14

First we translate this formula into our language. The four immersions $K_{+}$, $K_{-}, K_{0}, K_{\infty}$, which agree everywhere near a single crossing, are shown in Figure 14.

Let us consider the singular knot $K:=K\left(x_{1}, \ldots, x_{i}+\frac{1}{2}, \ldots, x_{e}, x_{f}\right)$. It is easy to see that $K_{+}=\mathcal{K}\left(x_{1}, \ldots, x_{i}+1, \ldots, x_{e}, x_{f}\right)$ and $K_{-}=\mathcal{K}\left(x_{1}, \ldots, x_{i}, \ldots, x_{e}, x_{f}\right)$. We already encountered $K_{0}$ in the proof of Theorem 3.3. It follows that $\lambda=$ $\Delta_{x_{i}} v_{2}\left(\mathcal{K}\left(x_{1}, \ldots, x_{i}, \ldots, x_{e}, x_{f}\right)\right)=$

$$
-x_{i}^{2}+\left(\alpha_{i}+\delta_{i} x_{f}\right) x_{i}+c_{i 1} x_{1}+\ldots+\widehat{c_{i i} x_{i}}+\ldots+c_{i e} x_{e}+\mu_{i} .
$$

For $K_{\infty}$ the situation is somewhat more difficult, but with the help of the map $\mathcal{K}$ we will be able to describe $K_{\infty}$. We define $\mathcal{K}^{s_{i}}$ as before but, instead of the curve $s$, we now use the curve $s_{i}$ obtained from the curve $s$ by making an $\infty$-splice at the double point $P_{i}$. The double points $P_{1}, \ldots, \widehat{P}_{i}, \ldots, P_{e}$ of $s_{i}$ have the same names as those of $s$. The orientation is chosen in such a way that $\mathrm{s}$ and $s_{i}$ run through $P_{f}$ in the same sense. There are two possibilities for the orientations of the branches in $D_{i}$, see Figure 15:
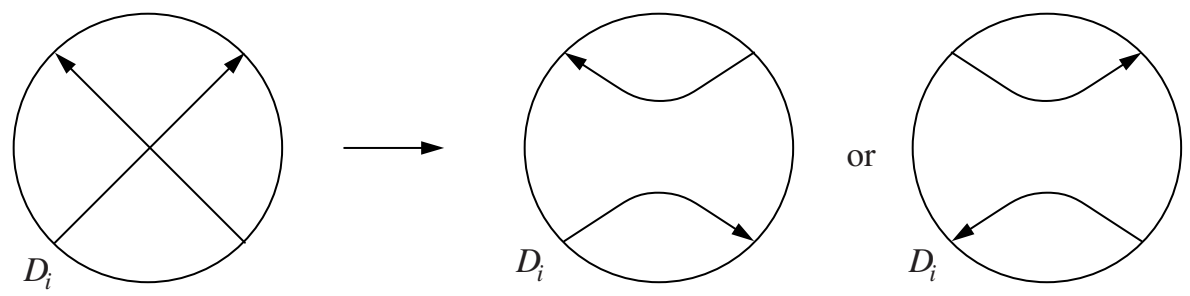

Figure 15

We shade two of the four local regions at any double point as shown in Figure 16 and compare these shadings of $s$ and $s_{i}$ in the example shown in Figure 17.

For $j \in\{1, \ldots, e\} \backslash\{i\}$, we define $\overline{x_{j}}$ as $x_{j}$ if the two shadings in $P_{j}$ coincide and as $1-x_{j}$, if they are opposite. A good approximation for $K_{\infty}$ is given by

$$
\mathcal{K}^{s_{i}}\left(\overline{x_{1}}, \ldots, \widehat{x_{i}}, \ldots, \overline{x_{e}}, x_{f}\right),
$$




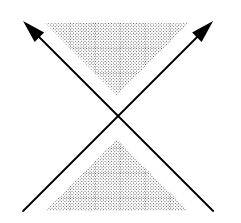

Figure 16

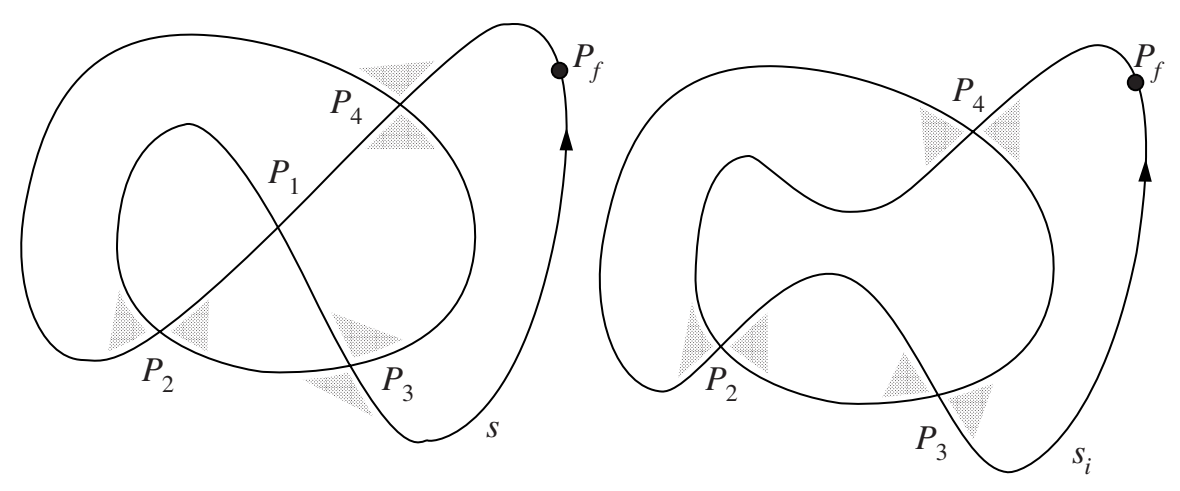

Figure 17

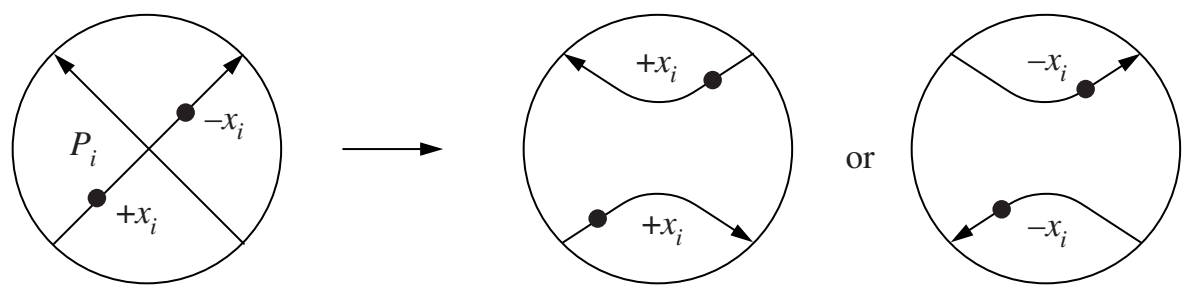

Figure 18

but the effect of the fibre fusions indicated in Figure 18 has not yet been taken into account.

We can do $\pm 2 x_{i}$ more fibre fusions in $P_{f}$ and move them towards $D_{i}$ using the trick in Figure 9. We give an example for the same curve as before. In Figure 19 we indicate that we have to take the following entries for $\mathcal{K}^{s_{1}}$ to get $K_{\infty}$ :

$$
K_{\infty}=\mathcal{K}^{s_{1}}\left(\widehat{x_{1}}, x_{2}, 1-x_{3}+x_{1}, 1-x_{4}+x_{1}, x_{f}+2 x_{1}\right) .
$$

With this choice, the gleams of corresponding regions on the left and right hand side coincide, see Figure 19.

Now we are ready to prove the theorem for $n=3$. For $i \in\{1, \ldots, e\}$ we have

$\Delta_{x_{i}} u_{3}\left(\mathcal{K}\left(x_{1}, \ldots, x_{e}, x_{f}\right)\right)=u_{2}\left(\mathcal{K}\left(x_{1}, \ldots, x_{e}, x_{f}\right)\right)-u_{2}\left(K_{\infty}\right)+\frac{1+(3 \lambda)^{3}-(3 \lambda+1)^{3}}{3 !}$. 


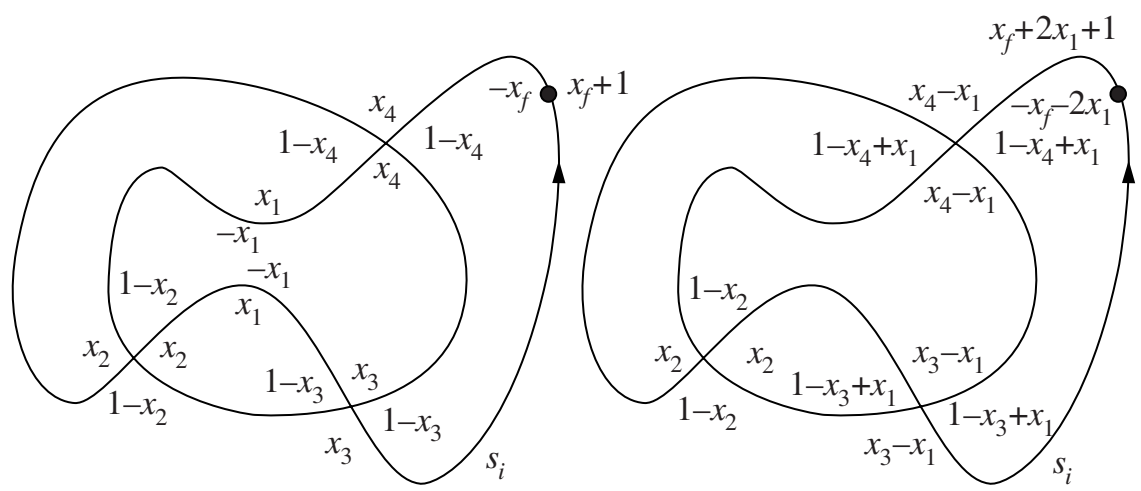

Figure 19

So $\Delta_{x_{i}}\left(u_{3} \circ \mathcal{K}\right)$ is a polynomial of degree $\leq 4$ in $x_{1}, \ldots, x_{f}$. We conclude that, up to an integration constant $G_{i}\left(x_{1}, \ldots, \widehat{x_{i}}, \ldots, x_{e}, x_{f}\right)$, the map $x_{i} \longmapsto\left(u_{3} \circ \mathcal{K}\right)$ is a polynomial of degree $\leq 5$ in $x_{1}, \ldots, x_{f}$. But this is true for all $i \in\{1, \ldots, e\}$, which implies that $u_{3} \circ \mathcal{K}$ is a polynomial of degree $\leq 5$ in $x_{1}, \ldots, x_{f}$ plus a function, let us say $g_{(3)}$, which depends on $x_{f}$ only. We choose $x_{1}, \ldots, x_{e} \in\{0,1\}$ such that the knot $\mathcal{K}\left(x_{1}, \ldots, x_{e}, 0\right)$ is trivial. For this choice of $x_{1}, \ldots, x_{e}$ we get: $\left(u_{3} \circ \mathcal{K}\right)\left(x_{1}, \ldots, x_{e}, x_{f}\right)=u_{3}\left(\mathcal{K}\left(x_{f}\right)\right)=$ a polynomial of degree $\leq 5$ in $x_{f}$ plus $g_{(3)}\left(x_{f}\right)$. This implies that $g_{(3)}$ is a polynomial of degree 6 in $x_{f}$, because we have shown in the first example of this section, that $u_{3}\left(\mathcal{K}\left(x_{f}\right)\right)$ is a polynomial of degree 6 in $x_{f}$.

Let us suppose now that for all $m \leq n$ the map $u_{m} \circ \mathcal{K}$ is a polynomial of degree $2 m$. We want to show that $u_{n+1} \circ \mathcal{K}$ is a polynomial of degree $2(n+1)$. Using the induction hypothesis and the result in Proposition 3.7 we see at once that for all $i \in\{1, \ldots, e\}$ the map $\Delta_{x_{i}}\left(u_{n+1} \circ \mathcal{K}\right)$ is a polynomial of degree $\leq 2 n$ in $x_{1}, \ldots, x_{f}$. This implies that $u_{n+1} \circ \mathcal{K}$ is a polynomial of degree $\leq 2 n+1$ in $x_{1}, \ldots, x_{f}$ plus a function, let us say $g_{(n+1)}$, which depends on $x_{f}$ only. We conclude, as above, that $g_{(n+1)}$ is a polynomial of degree $2(n+1)$ in $x_{f}$, which proves the theorem. $\square$

\section{Acknowledgements}

The author would like to thank his supervisor, Norbert A'Campo, for his patience. Thanks also go to Professor Turaev for his support and for helpful discussions. The author would also like to thank Andreas Meister and Ted Stanford who were patiently listening to his ideas. 


\section{References}

[B-L] J. S. Birman and X. S. Lin, Knot polynomials and Vassiliev's invariants, Invent. Math. 111 (1993), 225-270.

[G] M. N. Goussarov, Interdependent modifications of links and invariants of finite degree, Uppsala University preprint 1995:26.

[J] V. F. R. Jones, Hecke algebra representations of braid groups and link polynomials, Annals of Math. 126 (1987), 335-388.

[S] A. Shumakovitch, Shadow formula for the Vassiliev invariant of degree two, Uppsala University preprint 1995:27.

[T] V. G. Turaev, Shadow links and face models of statistical mechanics, Jour. of Differential Geometry, 36 (1992), 35-74.

Urs Burri

Mathematisches Institut

Universität Basel

Rheinsprung 21

4051 Basel

Switzerland

e-mail: burri@math.unibas.ch

(Received: April 30, 1996) 